\title{
Breast Feeding During Influenza Infection: An Issue for Developing Countries
}

\section{Viroj Wiwanitkit*}

Adjunct Professor, Joseph Ayobabalola University, Ikeji-Arakeji, Nigeria and Special Lecturer, Chulalongkorn University, Bangkok Thailand *Corresponding Author: Viroj Wiwanitkit, Adjunct Professor, Joseph Ayobabalola University, Ikeji-Arakeji, Nigeria and Special Lecturer, Chulalongkorn University, Bangkok Thailand.

Received: September 20, 2019; Published: October 10, 2019

DOI: $10.31080 /$ ASNH.2019.03.0497

\section{Abstract}

Influenza is an important respiratory viral infection. The infection is the present public health concern. In clinical nutrition, the important issue is on breast feeding during influenza infection. The recommendation for breast feeding during influenza is interesting in clinical nutrition. The present editorial discusses on this specific issue.

Keywords: Breastfeeding; Infection; Clinical Nutrition

\section{Introduction}

Influenza is an important respiratory viral infection. The infection can cause several respiratory problem. In severe case, serious pneumonias is possible and death might occur. The infection is the present public health concern. In clinical nutrition, the important issue is on breast feeding during influenza infection. The recommendation for breast feeding during influenza is interesting in clinical nutrition. The present editorial discusses on this specific issue.

What to be concerned for influenza and breastfeeding?

Breastfeeding is the way that the mother gives nutrition to her child. Influenza might occur during breastfeeding. The infection might attack either mother or child and there is a great consideration for the risk of breastfeeding. If mother or child get influenza, close contact can result in disease spreading. The mother can get infection from contact with her child. The child can also get infection due to contact with the mother. During breastfeeding the baby can be infected by mother's pathogens including to influenza virus due to contact with respiratory secretions or droplets [1]. In addition, use of drug for management of disease in mother is usually a big concern for drug transmission to child via breastmilk. Finally, there is an interesting concern whether influenza virus can be transmitted via breastmilk. There is still no study to determine whether virus is detectable in breastmilk of influenza mother or not. However, in animal model, parainfluenza virus can be detected in breastmilk [2].

\section{Recommendations: breastfeeding or not?}

Generally, breastfeeding is considered useful for promotion of good child health. Immunity to influenza is promoted via breastfeeding [4]. However, the advantage of breastfeeding during ma- ternal influenza infection has never been clarified. At present, pediatricians usually prefer to suggest the infected mother to give breastfeeding. This is still a big challenge.

There are many considerations. First, diagnosis of influenza is usually by clinical presumption. The definitive diagnosis is not generally performed in developing countries. There is a chance that the patient might have other serious infection. If the mother is infected with other infection, there is still the risk for possible transmission to the child via breastmilk. In addition, in case that both mother and child have influenza, it is evidenced that milk can interfere pharmacokinetics of antiviral drug oseltamivir [2]. The decreased efficacy of antiviral drug treatment in child can be expected. In addition, the two ways disease spreading between mother and child during breastfeeding might be expected. In general, if infected mother intend to perform breastfeeding, sanitation is important and it is usually not successful among the low educated mothers in developing countries. Therefore, some practitioners recommended temporary weaning of breastmilk and using supplementary milk product during influenza infection in either mother or child.

\section{Conflicts of Interest}

None.

\section{Bibliography}

1. Lanari M., et al. "Human milk, a concrete risk for infection?" Journal of Maternal-Fetal and Neonatal Medicine 25 (2012): 75-77.

2. Morimoto K., et al. "Effect of milk on the pharmacokinetics of oseltamivir in healthy volunteers". Journal of Pharmaceutical Sciences 100 (2011): 3854-3861. 
3. Kawakami Y., et al. "Infection of cattle with parainfluenza 3 virus with special reference to udder infection. I. Virus isolation from milk". Japanese Journal of Microbiology 10 (1996): 159-169.

4. Hanson LA. "Breastfeeding provides passive and likely longlasting active immunity". Annals of Allergy, Asthma and Immunology 81 (1998): 523-533.

Volume 3 Issue 11 November 2019

(C) All rights are reserved by Viroj Wiwanitkit. 